\title{
Thermodynamic Properties of Ammonia as an Ideal Gas*
}

\author{
Lester Haar
}

Instiłute for Basic Standards, National Bureau of Standards, Washington, D.C. 20234

(December 15, 1967)

\begin{abstract}
Thermodynamic functions for ammonia as an ideal gas at one atmosphere pressure have been evaluated. The contribution of the highly anharmonic out-of-plane vibrational mode, including its large coupling with rotation and its coupling with the other vibrational modes, is considered in detail. Tables of $C_{P}^{\circ} / R,\left(H^{\circ}-E_{0}^{\circ}\right) / R T,\left(E_{0}^{\circ}-G^{\circ}\right) / R T$, and $S^{\circ} / R$ have been calculated at closely spaced intervals from 50 to $5000^{\circ} \mathrm{K}$ within an overall uncertainty of less than 0.1 percent at $1000^{\circ} \mathrm{K}$.
\end{abstract}

Key Words: Ammonia, ideal gas, thermodynamic functions.

\section{Introduction}

This paper is part of a program originating with the Office of Standard Reference Data of the National Bureau of Standards to obtain accurate thermodynamic properties for ammonia. Subsequent papers of this program will include real-gas corrections to the ideal-gas properties contained herein.

In this paper the ideal-gas thermodynamic properties of ammonia in the ground electronic state are calculated at closely spaced temperature intervals from 50 to $5000{ }^{\circ} \mathrm{K}$. The calculations include the contributions of ordinary vibrational anharmonicity and vibrational-rotational coupling together with rotational stretching and rotational quantum effects. Particular attention is given to the treatment of the rotational and vibrational anharmonic effects due to the molecular inversion. To this end a method is developed for calculating this contribution such that an overall accuracy for the thermodynamic properties of ammonia approaching that of the simplest diatomic molecules is obtained.

In the vibrational ground state ammonia is a pyramidal molecule with the nitrogen atom approximately $0.38 \AA$ above the plane of the three hydrogen atoms. The distance between the nitrogen atom and each of the hydrogen atoms is approximately $1.0 \AA$. Thus the pyramid is relatively flat and the potential barrier to vibration of the nitrogen atom through the plane of the hydrogen atoms so as to invert the pyramid is low (approximately $\left.2000 \mathrm{~cm}^{-1}[1]\right) .^{1}$

Ammonia has six vibrational degrees of freedom. However, owing to symmetry there are only four normal vibrational modes, two of which have double degeneracies. The vibrational structure may thus be char-

\footnotetext{
*This work was supported in part by the Office of Standard Reference Data, NBS.
}

${ }^{1}$ Figures in brackets indicate the literature references at the end of this paper. acterized by the six quantum numbers $\left(v_{1} v_{2} v_{3}^{l_{3}} v_{4}^{l_{4}}\right)$, where the $v_{i}$ are the principal vibrational quantum numbers associated with the vibrational modes $\nu_{i}$ and the $l$ characterize the small coupling of the degenerate $\nu_{3}$ and $\nu_{4}$ asymmetric vibrational modes with the angular momentum of the molecule. The $\nu_{1}$ and $\nu_{2}$ modes are symmetric, nondegenerate vibrations corresponding to stretching the N-H bond and spreading the pyramid, respectively. (The $\nu_{2}$ mode is also referred to as the out-of-plane vibration.) The rotational structure for the ground state is that of a symmetric top, so that the rotational energy levels are characterized by the two quantum numbers $J$ and $K$.

The $\nu_{2}$ mode is the vibration associated with the inversion of the pyramid. Each vibrational state of this mode is split into two components, corresponding respectively to symmetric and antisymmetric eigenfunctions. (In the nomenclature these are identified by the superscript " $s$ " or " $a$ " to the quantum number $v_{2}$.) The spacing of these levels is highly anharmonic except for vibrational energies sufficiently higher or lower than the potential barrier to inversion. Thus the vibrational levels for the ground state of the $\nu_{2}$ mode are only slightly split, of the order of a fraction of a wavenumber, but the splitting increases rapidly with increasing vibrational quantum number. At energy levels sufficiently elevated above the barrier (greater than about $3000 \mathrm{~cm}^{-1}$ ) the levels become uniformly spaced with a spacing of approximately half that of the $\nu_{2}$ fundamental. Similarly, due to inversion the rotational structure undergoes a transition from that of a pyramid for the low values of the $v_{2}$ quantum number to that of a planar molecule for the higher values, so that the rotational motion is also strongly coupled to the $\nu_{2}$ mode. For a more detailed discussion of these effects, see the review by Herzberg [2] of the pyramidal $X Y_{3}$ molecule and Nielsen [3]. The energy structure 
of the other vibrational modes is similar to that of typical, slightly anharmonic rotating vibrators.

In section 2 the equations for the calculations are developed. This is followed in section 3 by a discussion of the molecular data, and in section 4 by a discussion of the calculated properties and an evaluation of their accuracy. Finally, a discussion is given in section 5 of some of the earlier work.

\section{Partition Function}

It is convenient to represent the rotational and vibrational structure by an expression for which the energy levels of the $\nu_{2}$ mode and its coupling with the other degrees of freedom are written explicitly. Thus the energy levels in wavenumbers are written

$$
T(v, J, K)=G^{\prime}(v)+F^{\prime}(v, J, K)+G_{2}(v, J, K),
$$

where the primes denote that dependence on $v_{2}$ is omitted. The first term in eq (1) describes the vibrational energy characterized by $\left(v_{1} 0 v_{3}^{l_{3}} v_{4}^{l_{4}}\right)$ and in notation similar to that in Herzberg [2] is approximated by

$$
\begin{gathered}
G^{\prime}(v)=\sum_{i}^{\prime} v_{i} v_{i}+\sum_{i>j}^{\prime} x_{i j} v_{i} v_{j}+\sum_{i}^{\prime} x_{i i}\left(v_{i}-1\right) v_{i}+\ldots \\
+g_{34} l_{3} l_{4}+g_{33}\left(l_{3}^{2}-v_{3}\right)+g_{44}\left(l_{4}^{2}-v_{4}\right)+\ldots \\
i, j \neq 2,
\end{gathered}
$$

where the $\nu_{i}$ are fundamentals and the $x$ and $g$ are relatively small anharmonicities. The second term in eq ( 1 ) is approximated by

$$
\begin{aligned}
& F^{\prime}(v, J, K)=B_{v}^{\prime} J(J+1)+\left(C_{v}^{\prime}-B_{v}^{\prime}\right) K^{2} \\
& \quad-D_{v}^{J^{\prime}} J^{2}(J+1)^{2}-D_{v}^{J K^{\prime}} J(J+1) K^{2}-D_{v}^{K^{\prime}} K^{4}+\ldots,
\end{aligned}
$$

where $C_{v}$ is the rotational constant corresponding to rotation about the symmetry axis and $B_{v}$ is the constant for axes perpendicular to the symmetry axis. The quantities $D_{v}^{J}, D_{v}^{J K}$, and $D_{v}^{K}$ are small coefficients associated with centrifugal distortion of the molecule. The subscript $v$ to each of the coefficients of eq (3) indicate dependence or coupling with the vibrational motion. The primes indicate that dependence on $v_{2}$ is omitted; that is, the rotational constants of eq (3) are evaluated for $v_{2}$ equal to zero. The coupling of the rotational motion to vibrations other than the $\nu_{2}$ mode is relatively small and is included here only to first order in the fundamentals.

$$
\begin{aligned}
& B_{v}^{\prime}=B_{0}-\sum_{i}^{\prime} \alpha_{i}^{B} v_{i}+ \\
& C_{v}^{\prime}=C_{0}-\sum_{i}^{\prime} \alpha_{i}^{C} v_{i}+, \quad i \neq 2
\end{aligned}
$$

where $B_{0}$ and $C_{0}$ are rotational constants, and the $\alpha$ are small corrections. All coordinates involving the $\nu_{2}$ mode, including its coupling with the other degrees of freedom are included in $G_{2}$. Thus $G_{2}$ includes those effects characterized by

$$
\left(v_{1} v_{2} v_{3}^{l_{3}} v_{4}^{l_{4}} J K\right)-\left(v_{1} 0 v_{3}^{l_{3}} v_{4}^{l_{4}} J K\right) .
$$

The partition function for the rotational and vibrational structure may be written

$$
Q=\sum_{v, J, K} \exp \left\{-T(v, J, K) c_{2} / T\right\},
$$

where $T(v, J, K)$ is discussed in eqs (1), (2), and (3). $c_{2}$ is the second radiation constant and $T$ is the absolute temperature. The sum extends over all rotational and vibrational degrees of freedom including all values of the $v, J, K$ quantum numbers. In the evaluation of eq (4) the contributions of the vibrational anharmonicity and vibration-rotation coupling effects contained in eqs (2) and (3) are all to be accounted for to first order; that is, only terms linear in $x, g$, and $\alpha$ are retained. Though $G_{2}$ is not conveniently described by the usual power series in the vibrational quantum numbers, its contribution, including coupling effects with the other degrees of freedom, is also to be included to this order.

The contribution of the $G_{2}$ term is obtained by expanding the partition function about the term-byterm sum of the observed $\left(0 v_{2} 00\right)$ energy levels. This summation incorporates the major effect of the highly anharmonic $\nu_{2}$ mode, which is the coupling between the symmetric and antisymmetric states, but it does not include the contribution of the $\nu_{2}$ coupling with the other vibrational modes. From an examination of the spectroscopic vibrational data (see Benedict et al. [4]), it can be seen that this cross coupling produces a shifting of energy levels of about the same magnitude as that of the other vibrational couplings (those represented by the $x$ and $g$ terms in eq (2)). Thus the contribution to the partition function of this cross coupling should be of the same order as that from the other anharmonicities, and a first order approximation for this contribution should be adequate.

In the expansion that follows expressions for the cross coupling are developed and their contribution for each individual $\left(0 v_{2} 00\right)$ state obtained explicitly. The cross coupling between a pair of vibrational states, e.g., states corresponding to the particular quantum numbers $v_{1}$ and $v_{2}$, is defined as

$$
G_{v_{1} v_{2}} \equiv G\left(v_{1} v_{2} 00\right)-G\left(v_{1} 000\right)-G\left(0 v_{2} 00\right),
$$

where $G\left(v_{1} v_{2} v_{3}^{l_{3}} v_{4}^{l_{4}}\right)$ is the vibrational energy. A useful representation for the coupling involving the highly anharmonic $\nu_{2}$ mode is the development of $G_{v_{1} v_{2}}$ as a power series of products of the observed energies, that is

$$
G_{v_{1} v_{2}}=k_{12} G\left(v_{1} 000\right) \cdot G\left(0 v_{2} 00\right)+\ldots .
$$

The constant $k_{12}$ is readily determined in terms of the observed coupling. Thus for coupling between the fundamentals the observed coupling $x_{12}$ is given by 


$$
\begin{aligned}
x_{12} & =k_{12} G(1000) \cdot G(0100)+\ldots \\
& =k_{12} \nu_{1} \nu_{2}+\ldots .
\end{aligned}
$$

Note that eq (6) reduces identically to the power series of eq (2) when the cross coupling is between nearly harmonic modes.

Consider the energy of the nearly harmonic oscillator $\nu_{m}$, neglecting all anharmonicities but the cross coupling with $\nu_{2}$. The energy levels for such an oscillator are given by

$$
G_{m}=\nu_{m} v_{m}+G_{v_{m} v_{2}}
$$

Substituting from eq (6) and defining $E_{2} \equiv G\left(0 v_{2} 00\right)$, this becomes

$$
G_{m} \cong \nu_{m} v_{m}+k_{2 m} \nu_{m} v_{m} \cdot E_{2}
$$

The contribution of $G_{m}$ to the partition function is the factor

$Q_{m}=\left\{\sum_{v_{m}} \exp -\left[\left(c_{2} / T\right)\left(\nu_{m} v_{m}+k_{2 m} E_{2} \nu_{m} v_{m}\right)\right]\right\}^{d_{m}}$

where $d_{m}$ is the degeneracy of the $m$ th mode. The anharmonicity term in the exponential of eq (8) is now expanded and only the linear term retained to yield

$$
Q_{m}=\left\{\sum_{v_{m}}\left\{\exp \left[-\frac{c_{2}}{T} \nu_{m} v_{m}\right]\right\}\left\{1-\frac{c_{2}}{T} k_{2 m} \nu_{m} v_{m} E_{2}\right\}\right\}^{d_{m}} .
$$

Using the identity

$$
-\frac{d}{d \alpha} \sum_{v} e^{-\alpha v}=\sum_{v} v e^{-\alpha v}
$$

the contribution of $Q_{m}$ becomes

$$
Q_{m}=\left(1-e^{-\frac{c_{2}}{T} \nu_{m}}\right)^{-d_{m}}\left(1-f_{2 m}\right)
$$

where

$$
f_{2 m}=d_{m} \frac{c_{2}}{T} k_{2 m} E_{2} \nu_{m} /\left(e^{c_{2} \nu m / T}-1\right) .
$$

The factor $\left(1-f_{2 m}\right)$ is the contribution of the interaction of a particular $\left(0 v_{2} 00\right)$ energy state with the $m$ th vibrational mode, and it depends explicitly on $E_{2}$. It is clear then that the effect of this coupling is to modify each of the terms in the term-by-term sum over the $\left(0 v_{2} 00\right)$ energy levels by the factor $\left(1-f_{2 m}\right)$. The term-by-term sum including cross coupling with the other vibrational modes is thus given by

$$
Q_{v_{2}}(\mathrm{vib})=\sum_{E_{2}} \prod_{m}\left(1-f_{2 m}\right) \exp \left(-c_{2} E_{2} / T\right) .
$$

The prime indicates that the $m=2$ factor in the product corresponding to self coupling of the $\nu_{2}$ mode is omitted.

The coupling of rotation with the $\nu_{2}$ mode is included by evaluating the classical rotational partition function for each of the $\left(0 v_{2} 00\right)$ energy levels and employing these as separate weighting factors to each of the terms in eq (12). These factors are the usual expression for a classical nonrigid rotator,

$$
Q_{R}\left(v_{2}\right)=\pi^{1 / 2}\left(\sigma_{B}^{2} \sigma_{C}\right)^{-1 / 2}\left(1+\rho^{(1)} T+\rho^{(2)} T^{2}\right) .
$$

The quantities $\sigma_{B}$ and $\sigma_{C}$ are given by,

$$
\begin{gathered}
\sigma_{B}=\frac{c_{2}}{T} B_{v_{2}}, \\
\sigma_{C}=\frac{c_{2}}{T} C_{v_{2}},
\end{gathered}
$$

where $B_{v_{2}}$ and $C_{v_{2}}$ are the observed rotational constants for the particular $\left(0 v_{2} 00\right)$ energy level. The factor $1+\rho^{(1)} T+\rho^{(2)} T^{2}$ is the contribution of rotational stretching (see, for example (5 and 14)), where the $\rho$ are calculated from the rotational stretching coefficients that apply to the particular $\left(0 v_{2} 00\right)$ level.

One further simplification is required in order to evaluate the out-of-plane contribution. This is to obtain an approximation for the contribution of the energy levels corresponding to high values of the $v_{2}$ quantum number. Note that the $\nu_{2}$ mode is nearly harmonic for energies corresponding to the fourth quantum number and higher, so that the contribution of these levels may be replaced by the well-known expression for a slightly anharmonic rotating vibrator. Combining eqs (12) and (13) and including the closed form approximation for the contribution of the states corresponding to quantum numbers given by $v_{2}>3$ yields the expression for $\left(C_{2}(v, J, K)\right.$ used in this calculation.

$$
\begin{gathered}
Q_{v_{2}}=\left\{\sum_{v_{2}<4} \frac{B_{0}}{B_{E}}\left(\frac{C_{0}}{C_{E}}\right)^{1 / 2}\left(1+\rho^{(1)} T+\rho^{(2)} T^{2}\right) .\right. \\
\left.\prod_{j}^{\prime}\left(1-f_{2 j}\right) e^{-\frac{c_{2}}{T} E}\right\} \\
+\frac{\exp \left(-c_{2} E_{4} s / T\right)}{1-\exp \left(-c_{2} \nu_{2}^{\prime} / T\right)} \cdot \frac{B_{0}}{B_{4}}\left(\frac{C_{0}}{C_{4}}\right)^{1 / 2} . \\
\prod_{j}\left(1-\theta_{2 j}\right)\left(1+\phi_{2}\right)\left(1+\rho^{(1)} T\right),
\end{gathered}
$$

where $E \equiv E_{2}$ and the subscript to $E$ now refers to the particular $v_{2}$ quantum number. The quantity in the braces in eq (14) includes levels only up to $v_{2}=3^{a}$. Note that the rigid rotational contribution for the ground state has been factored from eq (14). In the second term the factor $\frac{\exp \left(-c_{2} E_{4} s / T\right)}{1-\exp \left(-c_{2} \nu_{2}^{\prime} / T\right)}$ is the harmonic oscillator contribution with $E_{4} s$ as the first level 
and $\nu_{2}^{\prime}$ the fundamental. The factor $1-\theta_{2 j}$ is the ordinary anharmonicity coupling with the $j$ th vibrational mode, where

$$
\begin{aligned}
\theta_{i j} & =\frac{\frac{c_{2}}{T} x_{i j} d_{i} d_{j}}{\left(\exp \left(c_{2} \nu_{i} / T\right)-1\right)\left(\exp \left(c_{2} \nu_{j} / T\right)-1\right)}, \\
\theta_{i i} & =\frac{\frac{c_{2}}{T}\left(x_{i i}-g_{i i} / 3\right) d_{i}\left(d_{i}+1\right)}{\left(\exp \left(c_{2} \nu_{i} / T\right)-1\right)^{2}} .
\end{aligned}
$$

The factor $1+\phi_{2}$ is the well-known contribution of rotation-vibration coupling, where

$$
\phi_{i}=d_{i}\left(\frac{\alpha_{i}^{B}}{B}+\frac{1}{2} \frac{\alpha_{i}^{C}}{C}\right)
$$

Details concerning the derivation of quantities in eqs (15) may be found in Friedman and Haar [6], Woolley [7], and Pennington and Kobe [8], for example.

The contributions to the partition function of the two remaining terms $G^{\prime}(v, J, K)$ and $F^{\prime}(v, J, K)$ are quite straightforward (see $[6,7,8])$. With these the complete partition function is finally obtained.

$$
\begin{array}{r}
Q=\frac{1}{S} \prod_{m=1}^{4}{ }^{\prime}\left(1-e^{-\frac{c_{2}}{T} \nu_{m}}\right)^{-d_{m}} \prod_{i \leqslant j}^{4}{ }^{\prime}\left(1-\theta_{i j}\right) \prod_{k=1}^{4}\left(1+\phi_{k}\right) \\
\left(\frac{\Pi}{\sigma_{B_{0}}^{2} \sigma_{C_{0}}}\right)^{1 / 2} \cdot Q_{v_{2}} \cdot Q_{\mathrm{tr}} \cdot Q_{q r} .
\end{array}
$$

As noted earlier the primes on the product summations indicate that factors containing the vibrational coordinate $v_{2}$ are omitted. The quantity $S$ is the symmetry number. For the usual symmetric top molecule this has the value three. However, each of the components of the nondegenerate but split $\left(0 v_{2} 00\right)$ levels have been counted with a statistical weight of unity, so that the factor $Q_{v_{2}}$ is larger by a factor of two. This error is corrected by setting $S=6$. (Incidentally $S=6$ corresponds to the symmetry for a planar configuration for $\mathrm{NH}_{3}$, and eq (16) might be thought of as an expansion about this planar configuration.) The product over $m$ is the harmonic oscillator contribution for the modes other than $\nu_{2}$; the products over $i j$ and over $k$ are the corresponding anharmonicity and rotation-vibration coupling contributions, respectively. The degeneracy coefficients $d$ are unity for $m=1,2$, and two for $m=3,4$. The $\theta_{i j}$ and $\phi_{k}$ are given by eq (15). $Q_{\mathrm{tr}}$ is the usual contribution of translation. Lastly, the factor $Q_{q r}$ is a correction for rotational quantum effects. It is very small at temperatures for which the vibrations are appreciably excited, so that it is not coupled to the $\nu_{2}$ mode. The contribution $Q_{q r}$ is given by Stripp and Kirkwood [9] for an asymmetric rotator, which for a symmetric top rotator reduces to

$$
Q_{q r}=1+\frac{\sigma_{B}}{3}\left(1-\frac{1}{4} \frac{\sigma_{B}}{\sigma_{C}}\right)+\frac{\sigma_{B}^{2}}{15}\left(1-\frac{3}{4} \frac{\sigma_{B}}{\sigma_{C}}+\frac{7}{32} \frac{\sigma_{B}^{2}}{\sigma_{C}^{2}}\right) .
$$

\section{Molecular Data}

The molecular data upon which the calculations are based are listed in tables 1 and 2 . Table 1 lists the data sensitive to the inversion coordinates and utilized in the term-by-term sum (the quantity in braces in eq (14)). The vibrational energies corresponding to $v_{2}=0,3$ are from [4]. The energies corresponding to $v_{2}=1,2$ are from Garing et al. [10]. The values listed for the rotational constants $B_{v}$ and $C_{v}$ corresponding to the $v_{2}=0$ states are from [4]; those corresponding to the $v_{2}=1,2$ states from [10]; and to the $v_{2}=3$ states from Benedict et al. [11]. The rotational stretching constants $D_{v}^{J}, D_{v}^{J K}, D_{v}^{K}$ are the average of the symmetric and anti-

\begin{tabular}{|c|c|c|c|c|c|c|c|c|}
\hline State & $0^{s}$ & $0^{a}$ & $1^{s}$ & $1^{a}$ & $2^{s}$ & $2^{a}$ & $3^{s}$ & $3^{a}$ \\
\hline$\nu_{2}$ & 0.0 & 0.793 & 932.41 & 968.04 & 1597.42 & 1882.16 & 2383.46 & 2895.48 \\
\hline$B_{v_{2}}$ & 9.9443 & 9.939 & 10.07 & 9.89 & 10.26 & 9.73 & 9.50 & 9.20 \\
\hline$C_{v_{2}}$ & 6.196 & 6.198 & 6.05 & 6.13 & 5.90 & 6.13 & 6.16 & 6.26 \\
\hline$D_{v_{2}}^{J}$ & \multicolumn{2}{|c|}{$8.1 \times 10^{-4}$} & \multicolumn{2}{|c|}{$8.9 \times 10^{-4}$} & \multicolumn{2}{|c|}{$8.9 \times 10^{-4}$} & \multicolumn{2}{|c|}{$-3.3 \times 10^{-4}$} \\
\hline$D_{v_{2}}^{J K}$ & \multicolumn{2}{|c|}{$-14.5 \times 10^{-4}$} & \multicolumn{2}{|c|}{$-17.5 \times 10^{-4}$} & \multicolumn{2}{|c|}{$-17.5 \times 10^{-4}$} & \multicolumn{2}{|c|}{$15.0 \times 10^{-4}$} \\
\hline$D_{v_{2}}^{K}$ & \multicolumn{2}{|c|}{$7.5 \times 10^{-4}$} & \multicolumn{2}{|c|}{$10.5 \times 10^{-4}$} & \multicolumn{2}{|c|}{$10.5 \times 10^{-4}$} & \multicolumn{2}{|c|}{$-11.3 \times 10^{-4}$} \\
\hline
\end{tabular}
symmetric values. The values for $v_{2}=0$ are from [4]; for $v_{2}=1$, from [10]; for $v_{2}=3$, from [11]; finally, data for $v_{2}=2$ were taken equal to the $v_{2}=1$ values.

\begin{tabular}{|c|c|c|c|}
\hline$\nu_{1}\left(\begin{array}{llll}1 & 0 & 0 & 0\end{array}\right)$ & $\nu_{2}^{\prime}=E_{4 a}-E_{4 s}$ & $\nu_{3}\left(\begin{array}{llll}0 & 0 & 1 & 0\end{array}\right)$ & $\nu_{4}\left(\begin{array}{llll}0 & 0 & 0 & 1\end{array}\right)$ \\
\hline 3323.06 & 475 & 3443.381 & 1627.77 \\
\hline$x_{11}=-30$ & $x_{22}=+9$ & $x_{33}=-18.5$ & $x_{44}=-8.8$ \\
\hline$x_{12}=+20.6$ & $x_{23}=+32.36$ & $x_{34}=-17.3$ & $g_{44}=+2.65$ \\
\hline$x_{13}=-92$ & $x_{24}=-10.7$ & & \\
\hline$x_{14}=-6.7$ & $B_{4}=9.35, \quad C_{4}=6.21$ & & \\
\hline$\alpha_{1}^{B}=0.135$ & $\alpha_{2}^{B}=+0.015$ & $\alpha_{3}^{B}=+0.176$ & $\alpha_{4}^{B}=-0.230$ \\
\hline$\alpha_{1}^{C}=.078$ & $\alpha_{2}^{C}=+0.098$ & $\alpha_{3}^{C}=-0.009$ & $\alpha_{4}^{C}=+0.066$ \\
\hline
\end{tabular}

TABLE 1. The out-of-plane molecular constants in $\mathrm{cm}^{-1}$, $\mathrm{v}_{2} \leqslant 3,\left(0 \mathrm{v}_{2} 00\right)$

TABLE 2. Vibrational, rotational and coupling constants in $\mathrm{cm}^{-1}$, see eqs (2), (3)

The remaining molecular data are given in table 2 . The vibrational fundamental $\nu_{1}$ is from Benedict et al. [12]; $\nu_{3}$ is from [11]; $\nu_{4}$ is from [10]. The constants $\nu_{2}^{\prime}$ and $x_{22}$ which correspond to the high energy, nearlyharmonic region of the $\nu_{2}$ mode are derived from the reported values for the $\left(04^{s} 00\right)$ and $\left(05^{s} 00\right)$ levels at $3330 \mathrm{~cm}^{-1}$ and $4315 \mathrm{~cm}^{-1}$ given by Walsh and Warsop [13] and from theoretical considerations that at high energies the spacing between the levels should become nearly equal to one-half the $(0100)$ value. Also pertaining to this region are the rotational constants $B_{4}$ and $C_{4}$ which are taken equal to the averages of the values for $v_{2}=3^{s}$ and $3^{a}$. The anharmonicity coefficients $x_{12}, x_{13}$, $x_{14}, x_{23}, x_{24}, x_{33}$, and $x_{34}$ are from [4], and the coefficients $x_{44}$ and $g_{44}$ are from [10]. The anharmonicity coefficient $x_{11}$ is estimated from data for similar 
stretching vibrations. The coupling constants $\alpha_{i}^{N}$ are from [4]. The smali coefficient $g_{33}$ is taken as zero.

The values used for the atomic masses are from Cameron and Wichers [35]. The values used for the other physical constants are given in [36].

\section{Thermodynamic Functions}

The thermodynamic properties of ammonia as an ideal gas at a pressure of one atmosphere are listed in table 3. These include the Gibbs (free) energy function, enthalpy function, heat capacity at constant pressure, and entropy tabulated in dimensionless units at closely spaced temperature intervals from 50 to $5000{ }^{\circ} \mathrm{K}$. Should it be desired to obtain the properties for a pressure of 1 bar $\left(10^{6}\right.$ dynes $\mathrm{cm}^{-2}$ or $\left.10^{5} \mathrm{Nm}^{-2}\right)$ the magnitude of the tabulated free energy and entropy values should be increased by 0.0132 . Conversion factors to other units in frequent use are given in table 4 . The entries in table 3 are truncated at four decimals (ex- cept for $C_{p}^{\circ} / R$ above $2500{ }^{\circ} \mathrm{K}$ which is truncated at three) but at best they are accurate to three decimals. It is felt that the additional significance could be useful in applications involving differences between the entries. The density of entries permits use of linear interpolation throughout the table with a resulting interpolation error smaller than the uncertainty in the tabulated values.

As indicated by the earlier discussion the present calculation, except for the out-of-plane vibrations, is based on an expansion about the harmonic oscillatorrigid rotator model. In this expansion only first order (linear) terms in the coupling between the various degrees of freedom are retained. In addition to the errors inherent in the derivation of the partition function, eq (16), there are also errors due to uncertainties in the molecular constants, errors which arise from certain resonances between several of the low-lying vibrational states, the effects of which are omitted, and lastly errors arising from omission of excited electronic states.

TABLE 3. Thermodynamic functions for ammonia

The tabulated values are spaced sufficiently close to permit linear interpolation between the entries with a resulting error less than their overall uncertainty. For convenience the values at 273.15 and $298.15^{\circ} \mathrm{K}$ have also been included.

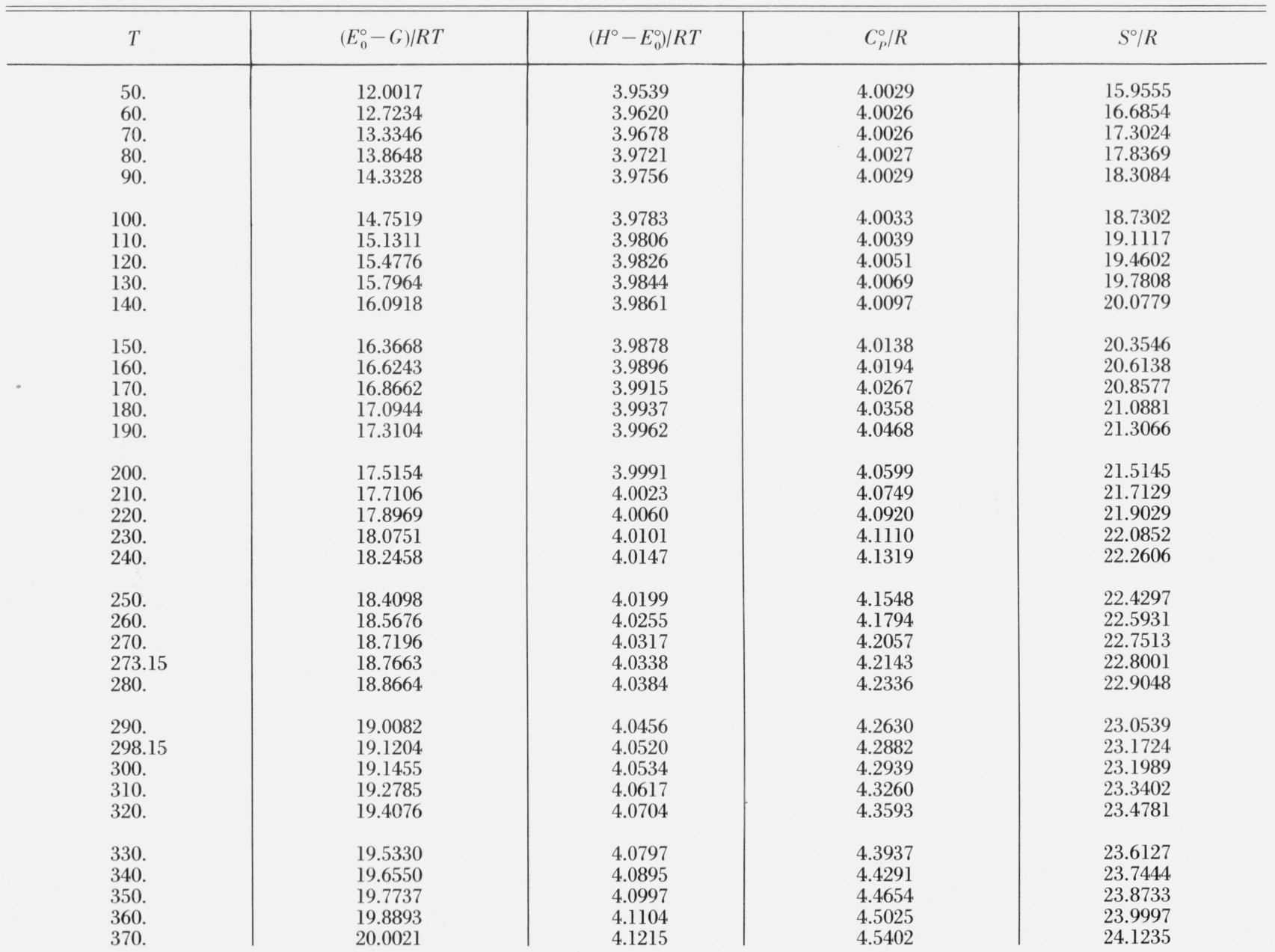


TABLE 3. Thermodynamic functions for ammonia-Continued

\begin{tabular}{|c|c|c|c|c|}
\hline$T$ & $\left(E_{0}^{\circ}-G\right) / R T$ & $\left(H^{\circ}-E_{0}^{\circ}\right) / R T$ & $C_{P}^{\circ} / R$ & $S^{\circ} / R$ \\
\hline $\begin{array}{l}380 . \\
390 . \\
400 . \\
410 . \\
420 .\end{array}$ & $\begin{array}{l}20.1121 \\
20.2196 \\
20.3247 \\
20.4275 \\
20.5282\end{array}$ & $\begin{array}{l}4.1330 \\
4.1449 \\
4.1572 \\
4.1699 \\
4.1829\end{array}$ & $\begin{array}{l}4.5785 \\
4.6173 \\
4.6565 \\
4.6961 \\
4.7359\end{array}$ & $\begin{array}{l}24.2451 \\
24.3646 \\
24.4819 \\
24.5974 \\
24.7111\end{array}$ \\
\hline $\begin{array}{l}430 . \\
440 . \\
450 . \\
460 . \\
470 .\end{array}$ & $\begin{array}{l}20.6268 \\
20.7234 \\
20.8181 \\
20.9111 \\
21.0024\end{array}$ & $\begin{array}{l}4.1962 \\
4.2098 \\
4.2237 \\
4.2379 \\
4.2524\end{array}$ & $\begin{array}{l}4.7759 \\
4.8161 \\
4.8563 \\
4.8966 \\
4.9368\end{array}$ & $\begin{array}{l}24.8230 \\
24.9332 \\
25.0419 \\
25.1491 \\
25.2548\end{array}$ \\
\hline $\begin{array}{l}480 . \\
490 . \\
500 . \\
520 . \\
540 .\end{array}$ & $\begin{array}{l}21.0921 \\
21.1802 \\
21.2669 \\
21.4360 \\
21.6000\end{array}$ & $\begin{array}{l}4.2671 \\
4.2820 \\
4.2971 \\
4.3278 \\
4.3593\end{array}$ & $\begin{array}{l}4.9771 \\
5.0172 \\
5.0572 \\
5.1368 \\
5.2158\end{array}$ & $\begin{array}{l}25.3592 \\
25.4622 \\
25.5640 \\
25.7639 \\
25.9592\end{array}$ \\
\hline $\begin{array}{l}560 . \\
580 . \\
600 . \\
620 . \\
640 .\end{array}$ & $\begin{array}{l}21.7591 \\
21.9137 \\
22.0643 \\
22.2109 \\
22.3540\end{array}$ & $\begin{array}{l}4.3912 \\
4.4237 \\
4.4566 \\
4.4898 \\
4.5232\end{array}$ & $\begin{array}{l}5.2939 \\
5.3712 \\
5.4475 \\
5.5230 \\
5.5975\end{array}$ & $\begin{array}{l}26.1503 \\
26.3374 \\
26.5208 \\
26.7007 \\
26.8772\end{array}$ \\
\hline $\begin{array}{l}660 . \\
680 . \\
700 . \\
720 . \\
740 .\end{array}$ & $\begin{array}{l}22.4937 \\
22.6302 \\
22.7638 \\
22.8946 \\
23.0227\end{array}$ & $\begin{array}{l}4.5569 \\
4.5907 \\
4.6247 \\
4.6588 \\
4.6929\end{array}$ & $\begin{array}{l}5.6711 \\
5.7438 \\
5.8157 \\
5.8867 \\
5.9568\end{array}$ & $\begin{array}{l}27.0506 \\
27.2210 \\
27.3885 \\
27.5533 \\
27.7156\end{array}$ \\
\hline $\begin{array}{l}760 . \\
780 . \\
800 . \\
820 \\
840\end{array}$ & $\begin{array}{l}23.1483 \\
23.2715 \\
23.3925 \\
23.5113 \\
23.6281\end{array}$ & $\begin{array}{l}4.7271 \\
4.7613 \\
4.7954 \\
4.8296 \\
4.8637\end{array}$ & $\begin{array}{l}6.0261 \\
6.0946 \\
6.1622 \\
6.2291 \\
6.2952\end{array}$ & $\begin{array}{l}27.8754 \\
28.0328 \\
28.1879 \\
28.3409 \\
28.4918\end{array}$ \\
\hline $\begin{array}{l}860 . \\
880 . \\
900 . \\
920 . \\
940 .\end{array}$ & $\begin{array}{l}23.7430 \\
23.8559 \\
23.9672 \\
24.0767 \\
24.1845\end{array}$ & $\begin{array}{l}4.8978 \\
4.9317 \\
4.9656 \\
4.9994 \\
5.0331\end{array}$ & $\begin{array}{l}6.3604 \\
6.4249 \\
6.4885 \\
6.5513 \\
6.6133\end{array}$ & $\begin{array}{l}28.6407 \\
28.7877 \\
28.9328 \\
29.0761 \\
29.2176\end{array}$ \\
\hline $\begin{array}{c}960 . \\
980 . \\
1000 . \\
1020 . \\
1040 .\end{array}$ & $\begin{array}{l}24.2909 \\
24.3957 \\
24.4990 \\
24.6010 \\
24.7017\end{array}$ & $\begin{array}{l}5.0667 \\
5.1001 \\
5.1334 \\
5.1665 \\
5.1995\end{array}$ & $\begin{array}{l}6.6745 \\
6.7348 \\
6.7943 \\
6.8530 \\
6.9108\end{array}$ & $\begin{array}{l}29.3575 \\
29.4958 \\
29.6324 \\
29.7676 \\
29.9012\end{array}$ \\
\hline $\begin{array}{l}1060 . \\
1080 . \\
1100 . \\
1120 . \\
1140 .\end{array}$ & $\begin{array}{l}24.8010 \\
24.8991 \\
24.9960 \\
25.0918 \\
25.1864\end{array}$ & $\begin{array}{l}5.2323 \\
5.2650 \\
5.2975 \\
5.3298 \\
5.3619\end{array}$ & $\begin{array}{l}6.9678 \\
7.0239 \\
7.0792 \\
7.1335 \\
7.1870\end{array}$ & $\begin{array}{l}30.0334 \\
30.1641 \\
30.2935 \\
30.4216 \\
30.5483\end{array}$ \\
\hline $\begin{array}{l}1160 . \\
1180 . \\
1200 . \\
1220 . \\
1240 .\end{array}$ & $\begin{array}{l}25.2799 \\
25.3724 \\
25.4639 \\
25.5543 \\
25.6438\end{array}$ & $\begin{array}{l}5.3938 \\
5.4256 \\
5.4571 \\
5.4884 \\
5.5195\end{array}$ & $\begin{array}{l}7.2397 \\
7.2914 \\
7.3424 \\
7.3924 \\
7.4416\end{array}$ & $\begin{array}{l}30.6738 \\
30.7980 \\
30.9209 \\
31.0427 \\
31.1633\end{array}$ \\
\hline $\begin{array}{l}1260 . \\
1280 . \\
1300 . \\
1320 . \\
1340 .\end{array}$ & $\begin{array}{l}25.7324 \\
25.8200 \\
25.9068 \\
25.9927 \\
26.0778\end{array}$ & $\begin{array}{l}5.5504 \\
5.5811 \\
5.6115 \\
5.6418 \\
5.6718\end{array}$ & $\begin{array}{l}7.4900 \\
7.5375 \\
7.5841 \\
7.6299 \\
7.6749\end{array}$ & $\begin{array}{l}31.2828 \\
31.4011 \\
31.5183 \\
31.6345 \\
31.7495\end{array}$ \\
\hline $\begin{array}{l}1360 . \\
1380 . \\
1400 . \\
1420 . \\
1440 .\end{array}$ & $\begin{array}{l}26.1620 \\
26.2455 \\
26.3281 \\
26.4101 \\
26.4912\end{array}$ & $\begin{array}{l}5.7016 \\
5.7311 \\
5.7604 \\
5.7895 \\
5.8184\end{array}$ & $\begin{array}{l}7.7190 \\
7.7624 \\
7.8049 \\
7.8466 \\
7.8875\end{array}$ & $\begin{array}{l}31.8636 \\
31.9766 \\
32.0886 \\
32.1996 \\
32.3096\end{array}$ \\
\hline
\end{tabular}


TABLE 3. Thermodynamic functions for ammonia-Continued

\begin{tabular}{|c|c|c|c|c|}
\hline$T$ & $\left(E_{0}^{\circ}-G\right) / R T$ & $\left(H^{\circ}-E_{0}^{\circ}\right) / R T$ & $C_{p}^{\circ} / R$ & $S^{\circ} / R$ \\
\hline $\begin{array}{l}1460 . \\
1480 . \\
1500 . \\
1550 . \\
1600 .\end{array}$ & $\begin{array}{l}26.5717 \\
26.6514 \\
26.7305 \\
26.9252 \\
27.1159\end{array}$ & $\begin{array}{l}5.8470 \\
5.8754 \\
5.9035 \\
5.9729 \\
6.0407\end{array}$ & $\begin{array}{l}7.9277 \\
7.9671 \\
8.0058 \\
8.0992 \\
8.1881\end{array}$ & $\begin{array}{l}32.4187 \\
32.5268 \\
32.6340 \\
32.8981 \\
33.1566\end{array}$ \\
\hline $\begin{array}{l}1650 . \\
1700 . \\
1750 . \\
1800 . \\
1850 .\end{array}$ & $\begin{array}{l}27.3028 \\
27.4861 \\
27.6659 \\
27.8424 \\
28.0158\end{array}$ & $\begin{array}{l}6.1071 \\
6.1720 \\
6.2354 \\
6.2974 \\
6.3579\end{array}$ & $\begin{array}{l}8.2728 \\
8.3532 \\
8.4298 \\
8.5027 \\
8.5719\end{array}$ & $\begin{array}{l}33.4099 \\
33.6581 \\
33.9013 \\
34.1398 \\
34.3737\end{array}$ \\
\hline $\begin{array}{l}1900 . \\
1950 . \\
2000 . \\
2050 . \\
2100 .\end{array}$ & $\begin{array}{l}28.1862 \\
28.3536 \\
28.5182 \\
28.6802 \\
28.8395\end{array}$ & $\begin{array}{l}6.4171 \\
6.4748 \\
6.5312 \\
6.5863 \\
6.6400\end{array}$ & $\begin{array}{l}8.6378 \\
8.7004 \\
8.7599 \\
8.8164 \\
8.8702\end{array}$ & $\begin{array}{l}34.6032 \\
34.8284 \\
35.0494 \\
35.2664 \\
35.4795\end{array}$ \\
\hline $\begin{array}{l}2150 . \\
2200 . \\
2250 . \\
2300 . \\
2350 .\end{array}$ & $\begin{array}{l}28.9964 \\
29.1508 \\
29.3030 \\
29.4528 \\
29.6005\end{array}$ & $\begin{array}{l}6.6925 \\
6.7437 \\
6.7937 \\
6.8425 \\
6.8901\end{array}$ & $\begin{array}{l}8.9214 \\
8.9701 \\
9.0162 \\
9.0602 \\
9.1021\end{array}$ & $\begin{array}{l}35.6889 \\
35.8945 \\
36.0966 \\
36.2953 \\
36.4906\end{array}$ \\
\hline $\begin{array}{l}2400 . \\
2450 . \\
2500 . \\
2550 . \\
2600 .\end{array}$ & $\begin{array}{l}29.7460 \\
29.8895 \\
30.0310 \\
30.1706 \\
30.3083\end{array}$ & $\begin{array}{l}6.9366 \\
6.9820 \\
7.0263 \\
7.0696 \\
7.1118\end{array}$ & $\begin{array}{l}9.1418 \\
9.1797 \\
9.216 \\
9.250 \\
9.282\end{array}$ & $\begin{array}{l}36.6826 \\
36.8715 \\
37.0573 \\
37.2402 \\
37.4201\end{array}$ \\
\hline $\begin{array}{l}2650 . \\
2700 . \\
2750 . \\
2800 . \\
2850 .\end{array}$ & $\begin{array}{l}30.4441 \\
30.5782 \\
30.7106 \\
30.8413 \\
30.9703\end{array}$ & $\begin{array}{l}7.1531 \\
7.1933 \\
7.2327 \\
7.2711 \\
7.3086\end{array}$ & $\begin{array}{l}9.313 \\
9.342 \\
9.370 \\
9.397 \\
9.422\end{array}$ & $\begin{array}{l}37.5972 \\
37.7716 \\
37.9432 \\
38.1123 \\
38.2789\end{array}$ \\
\hline $\begin{array}{l}2900 . \\
2950 . \\
3000 . \\
3100 . \\
3200 .\end{array}$ & $\begin{array}{l}31.0977 \\
31.2236 \\
31.3479 \\
31.5922 \\
31.8308\end{array}$ & $\begin{array}{l}7.3452 \\
7.3810 \\
7.4160 \\
7.4835 \\
7.5480\end{array}$ & $\begin{array}{l}9.445 \\
9.468 \\
9.490 \\
9.529 \\
9.564\end{array}$ & $\begin{array}{l}38.4429 \\
38.6046 \\
38.7639 \\
39.0757 \\
39.3788\end{array}$ \\
\hline $\begin{array}{l}3300 . \\
3400 . \\
3500 . \\
3600 . \\
3700 .\end{array}$ & $\begin{array}{l}32.0640 \\
32.2921 \\
32.5152 \\
32.7336 \\
32.9474\end{array}$ & $\begin{array}{l}7.6096 \\
7.6684 \\
7.7246 \\
7.7783 \\
7.8297\end{array}$ & $\begin{array}{l}9.596 \\
9.623 \\
9.648 \\
9.670 \\
9.688\end{array}$ & $\begin{array}{l}39.6736 \\
39.9605 \\
40.2398 \\
40.5119 \\
40.7771\end{array}$ \\
\hline $\begin{array}{l}3800 . \\
3900 . \\
4000 . \\
4100 . \\
4200 .\end{array}$ & $\begin{array}{l}33.1568 \\
33.3621 \\
33.5633 \\
33.7607 \\
33.9543\end{array}$ & $\begin{array}{l}7.8788 \\
7.9258 \\
7.9707 \\
8.0137 \\
8.0548\end{array}$ & $\begin{array}{l}9.704 \\
9.717 \\
9.728 \\
9.737 \\
9.743\end{array}$ & $\begin{array}{l}41.0357 \\
41.2879 \\
41.5341 \\
41.7744 \\
42.0091\end{array}$ \\
\hline $\begin{array}{l}4300 . \\
4400 . \\
4500 . \\
4600 . \\
4700 .\end{array}$ & $\begin{array}{l}34.1443 \\
34.3308 \\
34.5140 \\
34.6939 \\
34.8706\end{array}$ & $\begin{array}{l}8.0941 \\
8.1317 \\
8.1677 \\
8.2021 \\
8.2350\end{array}$ & $\begin{array}{l}9.748 \\
9.750 \\
9.751 \\
9.749 \\
9.746\end{array}$ & $\begin{array}{l}42.2384 \\
42.4626 \\
42.6817 \\
42.8960 \\
43.1056\end{array}$ \\
\hline $\begin{array}{l}4800 . \\
4900 . \\
5000 .\end{array}$ & $\begin{array}{l}35.0443 \\
35.2151 \\
35.3833\end{array}$ & $\begin{array}{l}8.2664 \\
8.2964 \\
8.3253\end{array}$ & $\begin{array}{l}9.741 \\
9.734 \\
9.725\end{array}$ & $\begin{array}{l}43.3107 \\
43.5115 \\
43.7086\end{array}$ \\
\hline
\end{tabular}

The expansion about the harmonic oscillator limit is essentially a low temperature expansion, and the p errors resulting therefrom for a particular vibrational mode are small provided $\frac{\nu}{T}$ is large, and are of the order of about the square of the linear term. The 


\begin{tabular}{|c|c|}
\hline $\begin{array}{c}\text { Conversion factors } \\
\text { to convert tabulated value } \\
\text { to quantity having dimensions } \\
\text { indicated below }\end{array}$ & Multiply by \\
\hline $\begin{array}{l}\text { Cal mole }{ }^{-1}{ }^{\circ} \mathrm{K}^{-1} \\
\text { Cal g-1 }{ }^{\circ} \mathrm{K}^{-1} \\
\text { Joules mole }{ }^{-1}{ }^{\circ} \mathrm{K}^{-1} \\
\text { Joules } \mathrm{g}^{-1}{ }^{\circ} \mathrm{K}^{-1}\end{array}$ & $\begin{array}{l}1.98717 \\
0.116682 \\
8.3143 \\
0.488197\end{array}$ \\
\hline
\end{tabular}

largest error of this type occurs for the $\nu_{4}$ mode. Thus for the most sensitive function, the heat capacity, the uncertainty at $2000^{\circ} \mathrm{K}$ is less than $10^{-2}$ dimensionless units, but increases to the order of 0.1 at $5000{ }^{\circ} \mathrm{K}$. The higher order anharmonicities would contribute an uncertainty perhaps as large. Though the anharmonicity coefficients $x_{11}$ and $x_{13}$ have large uncertainties, the errors introduced by these are mitigated by the fact that the fundamentals $\nu_{1}$ and $\nu_{3}$ are large. A 50 percent uncertainty in $x_{11}$ and $x_{13}$ results in an error of about 0.05 dimensionless units in $C_{P}^{\circ} / R$ at $5000{ }^{\circ} \mathrm{K}$. Setting $g_{33}$ equal to zero introduces negligible error since this coefficient introduces a small correction to an already small anharmonic contribution (owing to the large value of the $\nu_{3}$ fundamental). The remaining anharmonicities (other than the $x_{22}$ value in table 2) are probably accurate to better than several percent, and the uncertainties resulting from these are comparatively small. Uncertainties arising from errors in the rotational fundamentals are also comparatively small, as are uncertainties resulting from the use of estimates for some of the rotational stretching constants. The uncertainties that arise from errors in the rotationvibration coupling constants are perhaps half as large as those arising from errors in the anharmonicity coefficients.

The uncertainties in the values used for the fundamental frequencies are less than several tenths of a wavenumber, and the uncertainty from this source is negligible. Also negligible are the uncertainties due to errors in the observed overtones of the $\nu_{2}$ fundamental.

It is somewhat more difficult to determine the uncertainty resulting from estimates used for the molecular constants in the second term in eq (14). The largest uncertainty here arises from possible errors in $\nu_{2}^{\prime}$ and $E_{4^{*}}$. An uncertainty of several percent in these results in an uncertainty in $C_{P}^{\circ} / R$ of less than 0.002 dimensionless units at $1000{ }^{\circ} \mathrm{K}$, which increases to a maximum near $2000^{\circ} \mathrm{K}$ of less than 0.01 dimensionless units. The errors from estimates of the vibrational coupling constants used in this term are completely negligible below $2000^{\circ} \mathrm{K}$. Even if a 100 percent uncertainty in their contribution is assumed, the resulting uncertainty at $5000{ }^{\circ} \mathrm{K}$ still would be less than 0.1 dimensionless units in $C_{p}^{\circ} / R$.

The spectrum of ammonia is further complicated by several large resonances that occur between some of the low lying vibrational bands, such as the resonance between the combination bands $\left(2 \nu_{4}+\nu_{3}\right)$ and $\left(\nu_{1}+\nu_{3}\right)$ and between the bands $2 \nu_{4}$ and $\nu_{1}$ as discussed in [4]. Since the resonances tend to displace the respective bands symmetrically about their unperturbed values, it can be shown that the errors are less than the neglected higher order anharmonicities. A similar type of error occurs in the treatment of the coupling of the $\nu_{2}$ mode with the other modes. The coupling constants as reported are the average of the symmetric and antisymmetric values. Thus the effect of this approximation is to introduce errors similar to those arising from neglect of the resonances discussed above. The treatment of centrifugal distortion, which includes a quadratic term in the temperature, is consistent with the other approximations and the error from this source at $500{ }^{\circ} \mathrm{K}$ is probably less than 0.05 in $C_{P}^{\circ} / R$. At the low temperature end there is a small error arising from the rotational quantum approximation $Q_{\mathrm{qr}}$ (eq (17)). At $100^{\circ} \mathrm{K}$ this error is less than $10^{-3}$ in $C_{p}^{\circ} / R$, but is appreciably larger at $50{ }^{\circ} \mathrm{K}$.

Finally, the U.V. absorption spectrum indicates bands extending from $\sim 46,000 \mathrm{~cm}^{-1}$ to over 100,000 $\mathrm{cm}^{-1}$, as discussed, for example, in [13]. The error from omission of these even at the very highest temperatures considered here is completely negligible. It is felt, therefore, that the uncertainty in the tables in the temperature region 100 to $1000{ }^{\circ} \mathrm{K}$ is probably less than 0.003 dimensionless units in $C_{P}^{\circ} / R$. This uncertainty increases to 0.02 at $2000{ }^{\circ} \mathrm{K}$ and then increases very rapidly to perhaps 0.3 at $5000^{\circ} \mathrm{K}$. A more complete list of the uncertainty estimates, including those for the enthalpy and entropy, are given in table 5 .

TABLE 5. Estimates of uncertainty in dimensionless units

\begin{tabular}{c|c|c|c}
\hline \hline$T$ & $\Delta C_{P}^{\circ} / R$ & $\Delta\left(H^{\circ}-E_{0}^{\circ}\right) / R T$ & $\Delta S^{\circ} / R$ \\
\hline 100 & 0.003 & 0.002 & \\
1000 & .003 & .002 & .003 \\
2000 & .02 & .01 & .02 \\
3000 & .05 & .02 & .03 \\
4000 & .1 & .05 & .07 \\
5000 & .3 & .1 & .2 \\
\hline
\end{tabular}

\section{Discussion}

Ideal-gas calculations for $\mathrm{NH}_{3}$ have been reported in the literature since the early thirties, but many of these calculations were based on relatively incomplete and inaccurate molecular data and on simplified structural models. Also, for the most part, the temperature ranges were somewhat limited. The references [17-27] are representative of these early efforts.

Some of the more recent calculations which have been widely used include those of Harrison and Kobe [28] who calculated the heat capacity, enthalpy, and entropy from 273.16 to $1500{ }^{\circ} \mathrm{K}$. They included the major effects associated with the molecular inversion by summing the lower states of the $\nu_{2}$ mode term by term, but omitted all vibration and rotational coupling effects other than rotational stretching. The review by Davies reported in the monograph edited by Din [29] 
includes the specific heat, entropy, and enthalpy from 200 to $1000{ }^{\circ} \mathrm{K}$. The calculation follows essentially the procedure in [28], except that centrifugal stretching is omitted. Also it appears that the contribution of the states in the term-by-term sum of the $\nu_{2}$ mode was omitted above the level $3^{a}$. The most detailed of the previous calculations is that reported by Yungman et al. $[30]$, (which also appears in [16]), in which the free energy, entropy, and enthalpy are tabulated at $100{ }^{\circ} \mathrm{K}$ intervals from 298.15 to $6000{ }^{\circ} \mathrm{K}$. In addition to the term-by-term sum for the $\nu_{2}$ mode, this work also includes several of the first order vibrational and rotational coupling contributions. It omits, however, the contributions due to coupling of the $\nu_{2}$ mode with the other vibrational modes, and includes a somewhat simplified coupling of the $\nu_{2}$ mode with rotation. These calculations are based on essentially the same molecular data as those reported here, except that the values used for the vibrational coupling constants $x_{13}$ and $x_{44}$ are about twice as large. One of the most widely used set of tables is that of JANAF [31]. The reference refers to the latest revision for ammonia, September 30 , 1965. This calculation follows the procedures of [28] and [30], though the specific details are not given in the text. Finally, there are several recent calculations based on somewhat simplified molecular models, which are included in references [32-34].

The figures 1 and 2 include a comparison of some of the previous calculations with those made here. The ordinates are the deviations from the present calculations, that is the present calculation minus the others. Figure 1 is a plot of entropy differences, and figure 2 is a plot of heat capacity differences. It is most interesting to compare the data of Yungman et al. [30],

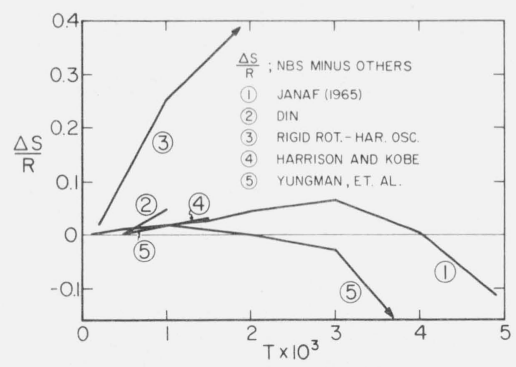

Figure 1. Dimensionless entropy differences.

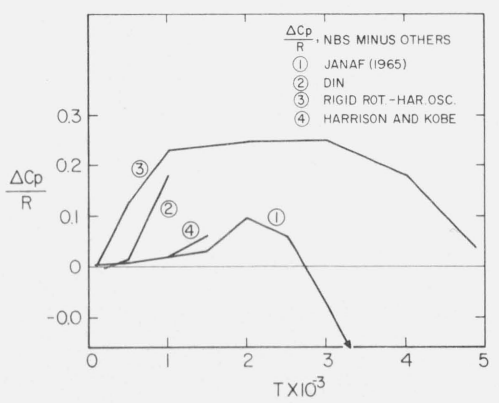

FigurE 2. Dimensionless heat capacity differences. curve \#5 in figure 1. At the low temperatures the agreement is quite good, but as the temperature is increased, a positive deviation occurs until about $3000^{\circ} \mathrm{K}$, when the deviation becomes strongly negative. The difference at the lower temperatures is probably due to their simplified treatment of the coupling of the $\nu_{2}$ mode with rotation. At temperatures above $1000^{\circ} \mathrm{K}$, the dominant contribution to the difference is from the $x_{24}$ term omitted in [30] which yields a positive contribution. At temperatures above $3000{ }^{\circ} \mathrm{K}$, the dominant contribution is from the omitted $x_{12}$ and $x_{23}$ terms which yield large negative contributions. In addition to these, the high value they used for $x_{44}$ (larger by a factor of two) tends to reduce the differences at temperatures below $3000{ }^{\circ} \mathrm{K}$, but magnifies them above. The same general comments apply to the JANAF [31] comparison as seen in curve \#l, except that the difference goes negative at somewhat higher temperatures and the positive deviations are considerably larger. Incidentally, the JANAF and Harrison and Kobe [28] (curve \#4) calculations are nearly identical for temperatures up to $1000{ }^{\circ} \mathrm{K}$. Curve $\# 3$ applies to a modified rigid rotator harmonic oscillator calculation as given in an older (now superseded) JANAF table. The comparison for curve \#2 [29] illustrates the sensitivity to the omission of the hierarchy of states corresponding to large $v_{2}$ quantum numbers $\left(v_{2}>3\right)$. The differences plotted in figure 2 exhibit essentially the same behavior as those in figure 1 except that they are somewhat larger.

It is a pleasure to thank Joseph Hilsenrath for many helpful technical discussions, particularly relating to the use of OMNITAB to program the calculation. In this latter connection, I also wish to thank Robert C. Thompson.

\section{References}

[1] J. D. Swalen and J. A. Ibers, J. Chem. Phys. 36, 1914 (1962).

[2] G. Herzberg, Infrared and Raman Spectra of Polyatomic Molecules, D. Van Nostrand \& Co., New York (1945).

[3] H. H. Nielsen, Revs. Modern Phys. 23, 90 (1951).

[4] W. S. Benedict and E. K. Plyler, Can. J. Phys. 35, 1235 (1957).

[5] R. S. McDowell, J. Chem. Phys. 39, 526 (1963).

[6] A. S. Friedman and L. Haar, J. Chem. Phys. 22, 2051 (1954).

[7] H. W. Woolley, J. Res. NBS 56, 105 (1956).

[8] R. E. Pennington and K. A. Kobe, J. Chem. Phys. 22, 1442 (1954).

[9] K. F. Stripp and J. G. Kirkwood, J. Chem. Phys. 19, 1131 (1951).

[10] J. S. Garing, H. H. Nielsen, and K. N. Rao, J. Mol. Spect. 3, 496 (1959)

[11] W. S. Benedict, E. K. Plyler, and E. D. Tidwell, J. Chem. Phys. 29, 829 (1958)

[12] W. S. Benedict, E. K. Plyler, and E. D. Tidwell, J. Chem. Phys. 32, 32 (1960).

[13] A. D. Walsh and P. A. Warsop, Trans. Faraday Soc. 57, 345 (1961).

[14] Y. S. Yungman, Zhur. Fiz. Khim. 35, 319 (1961).

[15] I. N. Godnev, Zhur. Fiz. Khim. 27, 1702 (1953).

[16] V. P. Glushko, Ed., Termodinamicheskie Svoistva Individual'nykh Vestchestv, II, Acad. Sci., USSR, Inst. of Applied Chemistry, Moscow (1962).

[17] D. S. Villars, Phys. Rev. 38, 1552 (1931); J. Am. Chem. Soc. 53, 2006 (1931).

[18] M. Trautz, Ann. Physik 9, 465 (1931). 
[19] F. A. F. Schmidt, Forsch. Arb. Ing. Wes. 8, 91 (1937)

[20] R. Overstreet and W. F. Giauque, J. Am. Chem. Soc. 59, 254 (1937).

[21] K. K. Kelley, U.S. Bur. Mines, Bull. 407 (1937).

[22] E. Justi, Spez-Wärme, Enthalpie und Dissociation Technischer Gase, J. Springer, Berlin (1938).

[23] C. C. Stephenson and H. O. McMahan, J. Am. Chem. Soc. 61, 437 (1939).

[24] K. S. Pitzer, J. Chem. Phys. 7, 251 (1939).

[25] R. F. Haupt and E. Teller, J. Chem. Phys. 7, 925 (1939).

[26] H. Zeise, Z. Elektrochem. 47, 380 (1941).

[27] H. W. Thomson, Trans. Faraday Soc. 37, 344 (1941).

[28] R. H. Harrison and K. A. Kobe, Chem. Eng. Progress 49, 351 (1953).

[29] F. Din, Thermodynamic Functions of Gases 1 (Butterworths, London, 1956).
[30] Y. S. Yungman, L. V. Gurvich, and N. P. Rtischeva, Tr. Gos. Inst. Prikl. Khim. 49, 26 (1962).

[31] JANAF Thermochemical Tables, Distrib. by Clearinghouse for Federal Scientific Information, PB168370-1, Sept. 30, 1965.

[32] C. L. Mader, Ideal Gas Thermodynamics of Detonation Products, U.S. A.E.C., AECU-4508 (1959).

[33] V. N. Khlebnikova and V. P. Morozov, Ukrain. Khim. Zhur. 24, 3 (1958).

[34] S. Sundaram, F. Suszek, and F. F. Cleveland, J. Chem. Phys. 32, 251 (1960).

[35] A. E. Cameron and E. Wichers, J. Am. Chem. Soc. 84, 4192 (1962).

[36] NBS Tech. News Bull. 47, No. 10 (Oct. 1963).

(Paper 72A2-495). 